\title{
Thermodynamics and thin film deposition of $\mathrm{MgB}_{2}$ superconductors
}

\author{
X X Xi ${ }^{1}$, X H Zeng ${ }^{1}$, A Soukiassian ${ }^{1}$, J Jones ${ }^{1}$, J Hotchkiss ${ }^{1}$, \\ Yu Zhong' ${ }^{2}$ C O Brubaker ${ }^{2}$, Zi-Kui Liu ${ }^{2}$, J Lettieri ${ }^{2}$, D G Schlom ${ }^{2}$,

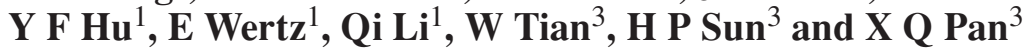 \\ ${ }^{1}$ Department of Physics, The Pennsylvania State University, University Park, PA 16802, USA \\ ${ }^{2}$ Department of Materials Science and Engineering, The Pennsylvania State University, \\ University Park, PA 16802, USA \\ ${ }^{3}$ Department of Materials Science and Engineering, The University of Michigan, Ann Arbor, \\ MI 48109, USA
}

Received 20 August 2001

Published 12 February 2002

Online at stacks.iop.org/SUST/15/451

\begin{abstract}
The recently discovered superconductor $\mathrm{MgB}_{2}$ with $T_{\mathrm{c}}$ at $39 \mathrm{~K}$ has great potential in superconducting microelectronics. Thermodynamics studies with the calculation of phase diagrams (CALPHAD) modelling technique show that due to the high volatility of $\mathrm{Mg}, \mathrm{MgB}_{2}$ is only thermodynamically stable under fairly high $\mathrm{Mg}$ overpressures for likely in situ growth temperatures. This provides a helpful insight into the appropriate processing conditions for $\mathrm{MgB}_{2}$ thin films, including the identification of the pressure-temperature region for adsorption-controlled growth. The initial $\mathrm{MgB}_{2}$ thin films were made by pulsed laser deposition followed by in situ annealing. The cross-sectional transmission electron microscopy reveals a nanocrystalline mixture of textured $\mathrm{MgO}$ and $\mathrm{MgB}_{2}$ with very small grain sizes. A zero-resistance transition temperature of $34 \mathrm{~K}$ and a zero-field critical current density of $1.3 \times 10^{6} \mathrm{~A} \mathrm{~cm}^{-2}$ were obtained. The qualities of these films are limited by the thermodynamic stability conditions, which favour deposition techniques that can maintain a high flux of $\mathrm{Mg}$.
\end{abstract}

\section{Introduction}

The recent discovery of superconductivity in $\mathrm{MgB}_{2}$ at $39 \mathrm{~K}$ has generated great interest $[1,2] . \mathrm{MgB}_{2}$ has the highest $T_{\mathrm{c}}$ known for non-oxide compounds. It has been shown that $\mathrm{MgB}_{2}$ is a phonon-mediated BCS superconductor [3] with an energy gap of $5.2 \mathrm{meV}$ at $4.2 \mathrm{~K} \mathrm{[4]} \mathrm{and} \mathrm{a} \mathrm{coherence} \mathrm{length} \mathrm{of}$ $50 \AA$ [5]. Its grain boundaries do not have a large detrimental effect on the superconducting current transport $[6,7]$. These properties hold tremendous promise for current-carrying largescale applications in wires and tapes. They also suggest that Josephson junctions of $\mathrm{MgB}_{2}$ may be much easier to fabricate than those made from the high temperature superconductors, which have much shorter coherence lengths. Such junctions could have the performance of conventional superconductor junctions, such as $\mathrm{Nb}$ and $\mathrm{NbN}$, but operate at a much higher temperature.

A $\mathrm{MgB}_{2}$ film processing technique compatible with multilayer depositions is needed for Josephson junction applications. Currently, two main types of deposition processes have been used, both involving annealing of films deposited at low temperatures. The first type employs ex situ annealing of low-temperature deposited $\mathrm{B}$ or $\mathrm{Mg}+\mathrm{B}$ films at $900{ }^{\circ} \mathrm{C}$ in $\mathrm{Mg}$ vapour. The resultant films exhibit bulk-like $T_{\mathrm{c} 0} \sim 39 \mathrm{~K}[8-10]$ and extremely high critical current density $\left(\sim 10^{7} \mathrm{~A} \mathrm{~cm}^{-2}\right.$ at low temperatures) $[11,12]$. However, the high-temperature ex situ annealing is unlikely to be compatible with multilayer device fabrications. The second type uses an in situ two-step process. Thin films or multilayers of $\mathrm{Mg}+\mathrm{B}$ or $\mathrm{Mg}+\mathrm{MgB}_{2}$ are deposited at low temperatures, and then annealed in situ in the deposition chamber at about $600{ }^{\circ} \mathrm{C}$ $[8,13-15]$. This process is potentially more compatible with junction fabrications, however the superconducting properties in such films are poorer than in the ex situ annealed films.

Due to the high volatility of $\mathrm{Mg}$, the fabrication of $\mathrm{MgB}_{2}$ thin films by in situ deposition is expected to be difficult. It has been demonstrated for numerous materials containing a volatile constituent that an understanding of 
the thermodynamics of the system can help identify the appropriate growth regime for these materials [16-20]. In this paper, we discuss a thermodynamic analysis of the $\mathrm{Mg}-\mathrm{B}$ system, which shows that the $\mathrm{MgB}_{2}$ phase is thermodynamically stable only under high $\mathrm{Mg}$ partial pressures. The result not only provides helpful insights into appropriate processing conditions for in situ deposition of $\mathrm{MgB}_{2}$ thin films, but also shows the limitation of the deposition technique involving in situ annealing. The result of $\mathrm{MgB}_{2}$ thin films made by an in situ process using pulsed laser deposition (PLD) is then presented. It shows that the superconducting properties of these films are weakened by the small $\mathrm{MgB}_{2}$ grain size of less than $50 \AA$.

\section{Thermodynamics of the Mg-B system}

Although $\mathrm{MgB}_{2}$ has been known and structurally characterized since the mid 1950s [21], no detailed thermodynamics information is available in the literature [22]. Our approach of the thermodynamic analysis is the calculation of phase diagrams (CALPHAD), a thermodynamic modelling technique using a computerized optimization procedure. In the CALPHAD approach, the Gibbs energies of individual phases in a system are constructed with models primarily based on the crystal structures of the phases [23]. For pure elements, the most commonly used model is that suggested by the Scientific Group Thermodata Europe (SGTE). The SGTE data used here are compiled by Dinsdale [24]. Using the experimentally measured enthalpy of formation and estimated decomposition temperatures, the Gibbs energy of each phase is evaluated with the Thermo-Calc program [25]. The phase equilibria are then calculated.

In the $\mathrm{Mg}-\mathrm{B}$ system, there are three intermediate compounds, $\mathrm{MgB}_{2}, \mathrm{MgB}_{4}$ and $\mathrm{MgB}_{7}$, in addition to gas and the solution phases, i.e. liquid, $h c p$ magnesium and $\beta$ rhombohedral boron [26]. The Gibbs energy of the gas phase assuming ideal mixing is

$$
G_{\mathrm{m}}=\sum y_{i}^{\circ} G_{i}+R T \sum y_{i} \ln y_{i}
$$

where $y_{i}$ and ${ }^{\circ} G_{i}$ are the mole fraction and Gibbs energy of species $i$ in the gas phase. The Gibbs energy of the solution phases treated as substitutional solutions is

$$
G_{\mathrm{m}}^{\Phi}=x_{\mathrm{Mg}}^{\circ} G_{\mathrm{Mg}}^{\Phi}+x_{\mathrm{B}}^{\circ} G_{\mathrm{B}}^{\Phi}+R T\left(x_{\mathrm{Mg}} \ln x_{\mathrm{Mg}}+x_{\mathrm{B}} \ln x_{\mathrm{B}}\right)
$$

where ${ }^{\circ} G_{i}^{\Phi}$ is the molar Gibbs energy of the pure element with the structure $\Phi$ from [24]. The Gibbs energies for the intermediate compounds are written using the two-sublattice model as

$$
G_{\mathrm{m}}^{\mathrm{MgB}_{x}}={ }^{\circ} G_{\mathrm{Mg}}^{\mathrm{hcp}}+x^{\circ} G_{\mathrm{B}}^{\beta-\text { rho }}+(1+x)\left(a^{\mathrm{MgB}_{x}}+b^{\mathrm{MgB}_{x}} T\right)
$$

where ${ }^{\circ} G_{\mathrm{Mg}}^{\text {hcp }}$ and ${ }^{\circ} G_{\mathrm{B}}^{\beta-\text { rho }}$ are the molar Gibbs energies of the $h c p \mathrm{Mg}$ and the $\beta$-rhombohedral $\mathrm{B}$, respectively, $a^{\mathrm{MgB}_{x}}$ and $b^{\mathrm{MgB}_{x}}$ are the parameters to be determined, and $\Delta G_{\mathrm{m}}^{\mathrm{MgB}_{x}}=$ $a^{\mathrm{MgB}_{x}}+b^{\mathrm{MgB}_{x}} T$ represents the Gibbs energy of formation of the compound $\mathrm{MgB}_{x}$ with $x$ being 2, 4 and 7, respectively, expressed in per mole of atoms.

Using the aforementioned procedure, the Gibbs energies of formation are obtained for the three compounds:
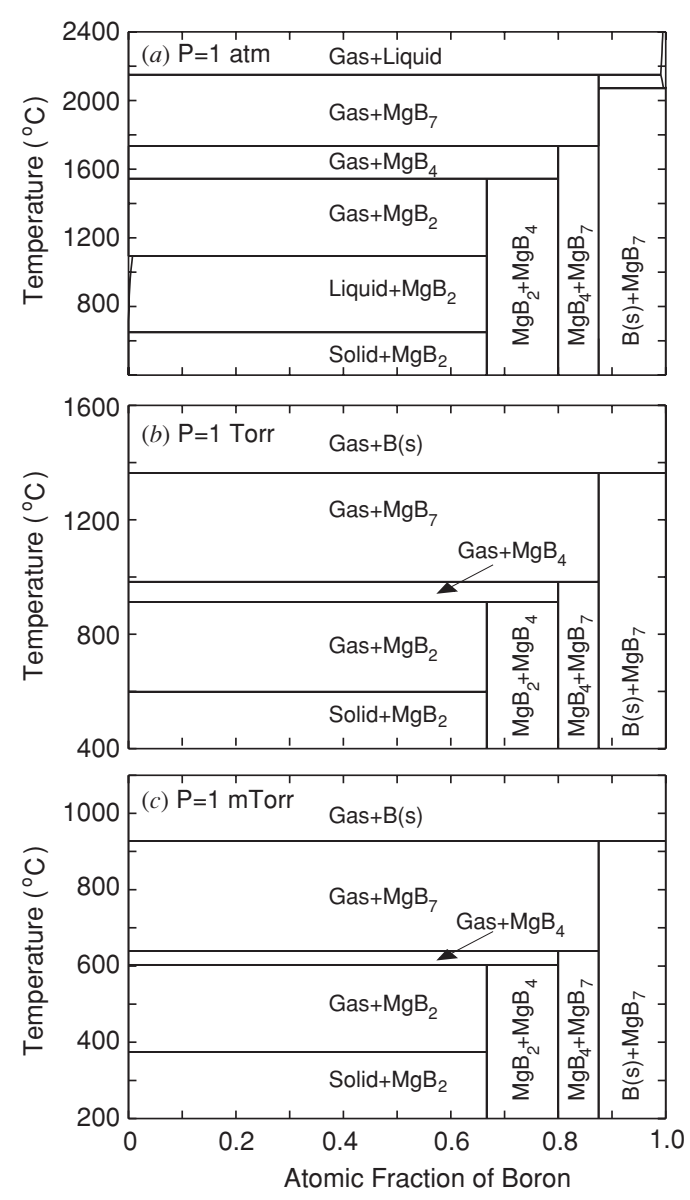

Figure 1. The temperature-composition phase diagrams of the $\mathrm{Mg}-\mathrm{B}$ system under the pressures of $(a) 1 \mathrm{~atm},(b) 1$ Torr and (c) 1 mTorr.

$\Delta G_{\mathrm{m}}^{\mathrm{MgB}_{2}}=-17121-3.815 T, \Delta G_{\mathrm{m}}^{\mathrm{MgB}_{4}}=-14441-2.458 T$ and $\Delta G_{\mathrm{m}}^{\mathrm{MgB}_{7}}=-13022-0.872 T$. From these results, binary phase diagrams can be calculated. In figure 1 the calculated temperature-composition phase diagrams for the $\mathrm{Mg}-\mathrm{B}$ system at (a) $1 \mathrm{~atm},(b) 1$ Torr and (c) 1 mTorr are plotted. The result for 1 atm pressure is consistent with the published $\mathrm{Mg}-\mathrm{B}$ phase diagram [22], which confirms the powerful capability of the CALPHAD technique. Above $1545{ }^{\circ} \mathrm{C} \mathrm{MgB}_{2}$ decomposes into a mixture of $\mathrm{MgB}_{4}$ and $\mathrm{Mg}$ vapour. When the pressure is reduced to 1 Torr, the phase diagram changes dramatically. Since the pressure is lower than the triple-point pressure of $\mathrm{Mg}\left(650{ }^{\circ} \mathrm{C}, 2.93\right.$ Torr $)$, the liquid phase of $\mathrm{Mg}$ disappears completely. The decomposition temperature of $\mathrm{MgB}_{2}$ decreases to $912{ }^{\circ} \mathrm{C}$. This temperature decreases further to $603{ }^{\circ} \mathrm{C}$ at a pressure of 1 mTorr. It is evident that $\mathrm{Mg}$ overpressure has a significant influence on the decomposition temperature of $\mathrm{MgB}_{2}$, which can be very low, thus severely limiting the thin film deposition temperature.

The kinetics of crystal growth require that an in situ film deposition process takes place at sufficiently high temperatures. The optimum temperature for epitaxial growth is typically about one half of the melting temperature, $T_{\mathrm{m}}$ (in Kelvin), although the minimum temperature can be much lower [27]. For example, the minimum epitaxial growth temperature for metals is about $T_{\mathrm{m}} / 8$ [28]. Thermodynamic 
calculation shows that $\mathrm{MgB}_{2}$ melts congruently at $2430{ }^{\circ} \mathrm{C}$ $(\sim 2700 \mathrm{~K})$ with an equilibrium vapour pressure exceeding 49000 Torr. Therefore, the optimum temperature for the deposition of epitaxial $\mathrm{MgB}_{2}$ films is around $\sim 1080{ }^{\circ} \mathrm{C}$ $(1350 \mathrm{~K})$. For $\mathrm{MgB}_{2}$ to be stable at $1080{ }^{\circ} \mathrm{C}$, a $\mathrm{Mg}$ partial pressure of at least 11 Torr is required. Converting the $\mathrm{Mg}$ partial pressure to $\mathrm{Mg}$ flux, $F$, from the deposition source using the formula $F=P / \sqrt{2 \pi m k_{\mathrm{B}} T}$, where $m$ is the mass of $\mathrm{Mg}$ atom [29, 30], 11 Torr is equivalent to an incident $\mathrm{Mg}$ flux of $2 \times 10^{21} \mathrm{Mg}$ atoms $/\left(\mathrm{cm}^{2} \mathrm{~s}\right)$, or a $\mathrm{Mg}$ deposition rate of $0.5 \mathrm{~mm} \mathrm{~s}^{-1}$ if all the $\mathrm{Mg}$ atoms stick and form a $\mathrm{Mg}$ layer with bulk density. This is impossible for most thin film deposition techniques.

Figure 1, however, illustrates the automatic composition control benefit that accompanies adsorption-controlled growth as is extensively used for III-V and II-VI compound semiconductors [31]. As long as the $\mathrm{Mg}$ : $\mathrm{B}$ ratio is above the 1:2 stoichiometry, any amount of extra $\mathrm{Mg}$ above this stoichiometric amount will vaporize and the desired $\mathrm{MgB}_{2}$ phase will result. The most critical requirement for controlling the stoichiometry is thus to avoid insufficient $\mathrm{Mg}$ supply, which will lead to $\mathrm{MgB}_{4}, \mathrm{MgB}_{7}$ or solid B phases.

From a thermodynamic perspective, deposition of a single-phase $\mathrm{MgB}_{2}$ film becomes easy when the growth conditions (substrate temperature and $\mathrm{Mg}$ overpressure) fall within a window where the thermodynamically stable phases are the desired $\mathrm{MgB}_{2}$ phase and gas phases. Within this growth window $\mathrm{MgB}_{2}$ does not decompose and excess $\mathrm{Mg}$ does not condense on the $\mathrm{MgB}_{2}$ surface, thus the formation of singlephase $\mathrm{MgB}_{2}$ is adsorption controlled and automatic. This window (the ' $\mathrm{Gas}+\mathrm{MgB}_{2}$ ' region) is best illustrated by the pressure-temperature phase diagram shown in figure 2. For a given deposition temperature, one can find the $\mathrm{Mg}$ partial pressure range to keep the $\mathrm{MgB}_{2}$ phase thermodynamically stable. As shown in the figure, this range extends over about three orders of magnitude. The boundaries of the growth window can be approximately expressed by the following equations: $\log (P)=-7561 / T+8.673$ (the upper boundary with solid $\mathrm{Mg}$ ) and $\log (P)=-10142 / T+8.562$ (the lower boundary with $\mathrm{MgB}_{4}$ ), where $P$ is in Torr and $T$ in Kelvin.

While the applicability of equilibrium thermodynamics to thin film growth has been established for many material systems, the non-equilibrium nature of specific deposition techniques can be quite important. For $\mathrm{MgB}_{2}$, a recent study has revealed a significant kinetic barrier to the thermal decomposition of $\mathrm{MgB}_{2}$ [32]. This opens the possibility of using non-equilibrium routes to drive the formation reaction of $\mathrm{MgB}_{2}$ to mitigate by as much as a factor of $10^{-3}$ the high $\mathrm{Mg}$ pressures described in this paper.

The implications of the thermodynamic analysis to the in situ $\mathrm{MgB}_{2}$ film deposition can be demonstrated by examining the necessary $\mathrm{Mg}$ overpressures for several possible deposition conditions. As discussed above, the optimum epitaxial growth temperature is $\sim 1080{ }^{\circ} \mathrm{C}$ for $\mathrm{MgB}_{2}$, which requires a $\mathrm{Mg}$ flux of $2 \times 10^{21} \mathrm{Mg}$ atoms $/\left(\mathrm{cm}^{2} \mathrm{~s}\right)$ or a $\mathrm{Mg}$ deposition rate of $0.5 \mathrm{~mm} \mathrm{~s}^{-1}$. The lower bound for epitaxial growth is unknown. For metals it is about $T_{\mathrm{m}} / 8$ [28], which would be $50{ }^{\circ} \mathrm{C}$ for $\mathrm{MgB}_{2}$. However, there has been no observation of $\mathrm{MgB}_{2}$ epitaxy for a deposition temperature lower than $400{ }^{\circ} \mathrm{C}$. From the phase diagram in

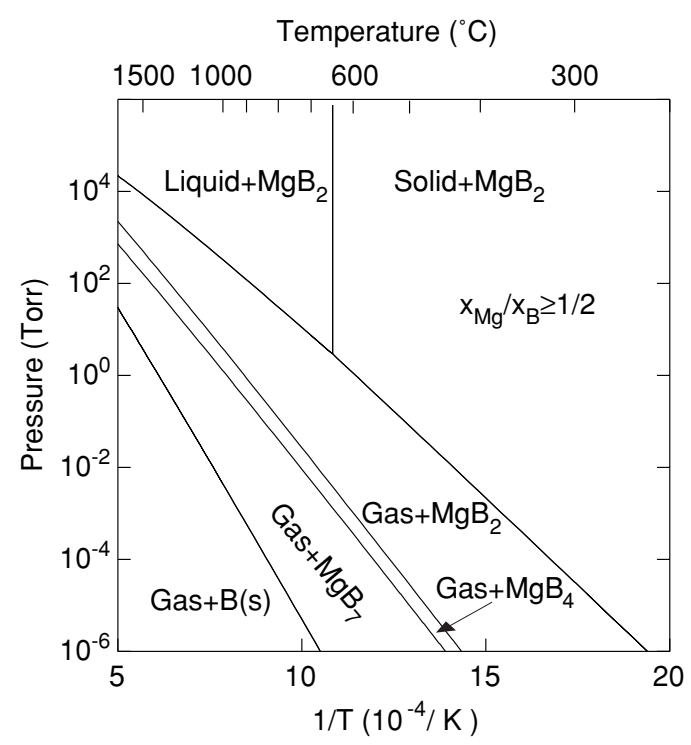

Figure 2. The pressure-temperature phase diagram for the $\mathrm{Mg}: \mathrm{B}$ atomic ratio $x_{\mathrm{Mg}} / x_{\mathrm{B}} \geqslant 1 / 2$. The region of ' $\mathrm{gas}+\mathrm{MgB}_{2}$ ' represents the thermodynamic stability window for the deposition of $\mathrm{MgB}_{2}$ thin films.

figure 2, a deposition temperature of $850{ }^{\circ} \mathrm{C}$ corresponds to a $\mathrm{Mg}$ overpressure of 340 mTorr, or a $\mathrm{Mg}$ flux of $6 \times 10^{19} \mathrm{Mg}$ atoms $/\left(\mathrm{cm}^{2} \mathrm{~s}\right)$ or a $\mathrm{Mg}$ deposition rate of $15 \mu \mathrm{m} \mathrm{s}^{-1}$. For $600{ }^{\circ} \mathrm{C}$, the necessary $\mathrm{Mg}$ overpressure is 0.9 mTorr, which corresponds to a $\mathrm{Mg}$ flux of $2 \times 10^{17} \mathrm{Mg}$ atoms $/\left(\mathrm{cm}^{2} \mathrm{~s}\right)$ or a $\mathrm{Mg}$ growth rate of $500 \AA \mathrm{s}^{-1}$. Clearly, these conditions favour deposition techniques that can maintain a high $\mathrm{Mg}$ overpressure during the deposition. This is further complicated by the oxygen contamination during the deposition in non-UHV systems. The Mg flux reacts with residual oxygen in the background, which effectively reduces the $\mathrm{Mg}$ overpressure thus pushing the system to the thermodynamically unstable region.

\section{Nanocrystalline $\mathrm{MgB}_{2}$ thin films by an in situ annealing process}

Before an in situ $\mathrm{MgB}_{2}$ thin film deposition technique that satisfies the thermodynamic stability conditions is developed, techniques involving post-deposition annealing are being investigated. We have used PLD with an in situ annealing procedure similar to those described by Blank et al [13], Christen et al [14] and Shinde et al [8], which is potentially more compatible with junction fabrications than the ex situ annealing process. The early reports from various groups on in situ $\mathrm{MgB}_{2}$ thin films show lower $T_{\mathrm{c} 0}$ around or below $25 \mathrm{~K}[8,13-15]$. Our result shows a much higher $T_{\mathrm{c} 0}$ of $34 \mathrm{~K}$. From the structural analysis, we find that our films are nanocrystalline and the small $\mathrm{MgB}_{2}$ size limits their superconducting properties.

The $\mathrm{MgB}_{2}$ films were deposited on (0001) $\mathrm{Al}_{2} \mathrm{O}_{3}$ substrates from targets prepared by pressing $\mathrm{Mg}$ powder with $\mathrm{MgB}_{2}$ powder at room temperature. The $\mathrm{Mg}: \mathrm{MgB}_{2}$ molar ratio was varied between $4: 1$ and 2:1. The films were deposited at 250-300 ${ }^{\circ} \mathrm{C}$ in an Ar atmosphere (99.999\% gas purity) of 120 mTorr. The background vacuum was in the low to mid 

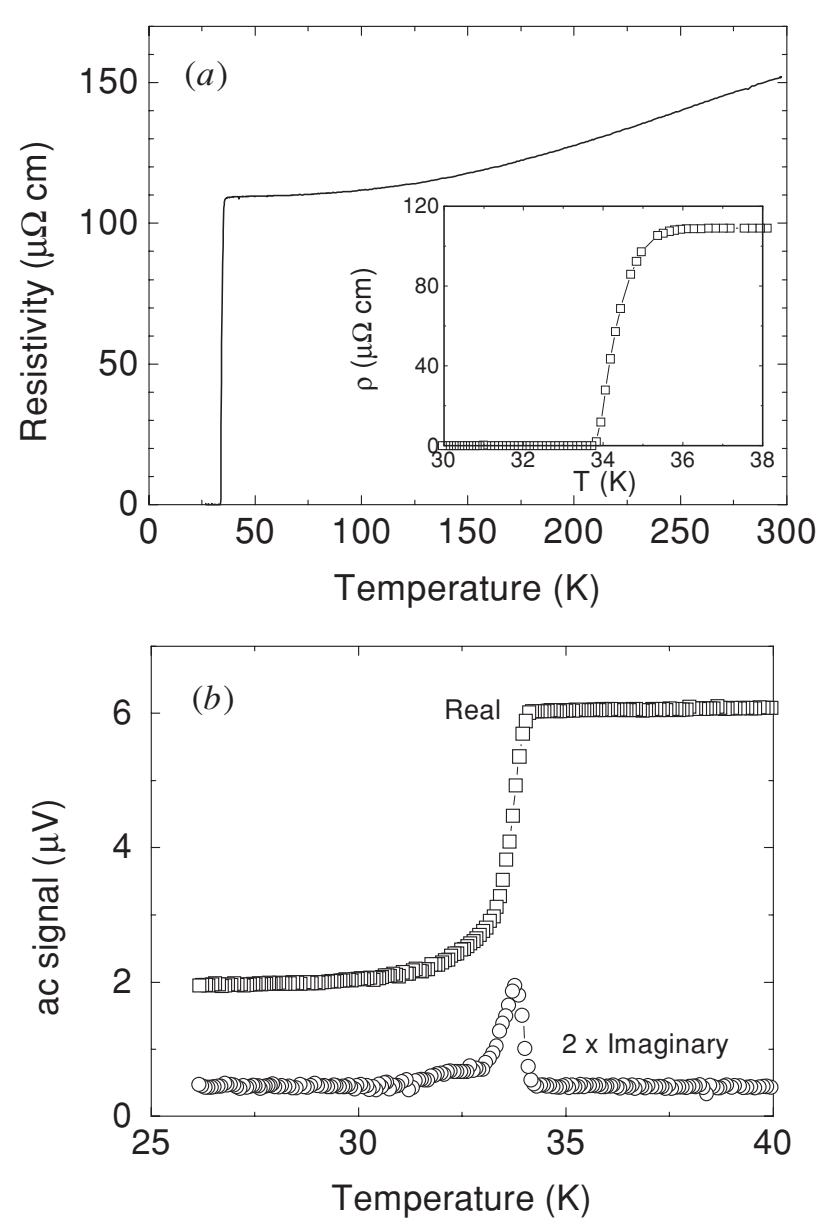

Figure 3. (a) Resistivity versus temperature curve for a $4000 \AA$ thick $\mathrm{MgB}_{2}$ film. (b) The ac susceptibility of the same film.

$10^{-7}$ Torr range. The energy density of the laser beam was $5 \mathrm{~J} \mathrm{~cm}^{-2}$ and the repetition rate was $5 \mathrm{~Hz}$. The deposited films were then heated at a rate of $40{ }^{\circ} \mathrm{C} \mathrm{min}-1$ to $630{ }^{\circ} \mathrm{C}$ and held there for $10 \mathrm{~min}$. The atmosphere during the heating and annealing was the same as during the deposition. After the in situ annealing, the films were cooled to room temperature in $\sim 20$ Torr Ar.

The film deposited at $250-300{ }^{\circ} \mathrm{C}$ was a likely mixture of $\mathrm{Mg}$ and amorphous $\mathrm{MgB}_{2}$ or $\mathrm{B}$. Several processes are involved in the in situ annealing: $\mathrm{Mg}$ evaporation, $\mathrm{MgB}_{2}$ phase formation, which is determined by the thermodynamics [33] and forward kinetics [32], nucleation and growth of crystallites, and $\mathrm{MgB}_{2}$ decomposition, which is determined by the thermodynamics [33] and a kinetic barrier [32]. High quality films are constrained by the balance of these processes, and a careful adjustment of the heating and annealing parameters such as temperature and duration is necessary.

In figure $3(a)$ we plot the resistivity versus temperature curve for a $4000 \AA$ thick $\mathrm{MgB}_{2}$ film. It shows a metallic behaviour with a residual resistance ratio, $\mathrm{RRR}=R(300 \mathrm{~K})$ / $R(40 \mathrm{~K})$, of 1.4 and the resistivity at room temperature is $\sim 150 \mu \Omega \mathrm{cm}$. Compared to high-density bulk samples, where $\mathrm{RRR}=25.3$ and $\rho(300 \mathrm{~K})=9.6 \mu \Omega \mathrm{cm}$ [34], the residual resistance ratio of the $\mathrm{MgB}_{2}$ film is much smaller and the resistivity much higher. This is likely to be due to the small grain sizes and existence of $\mathrm{MgO}$ in the film since precipitates
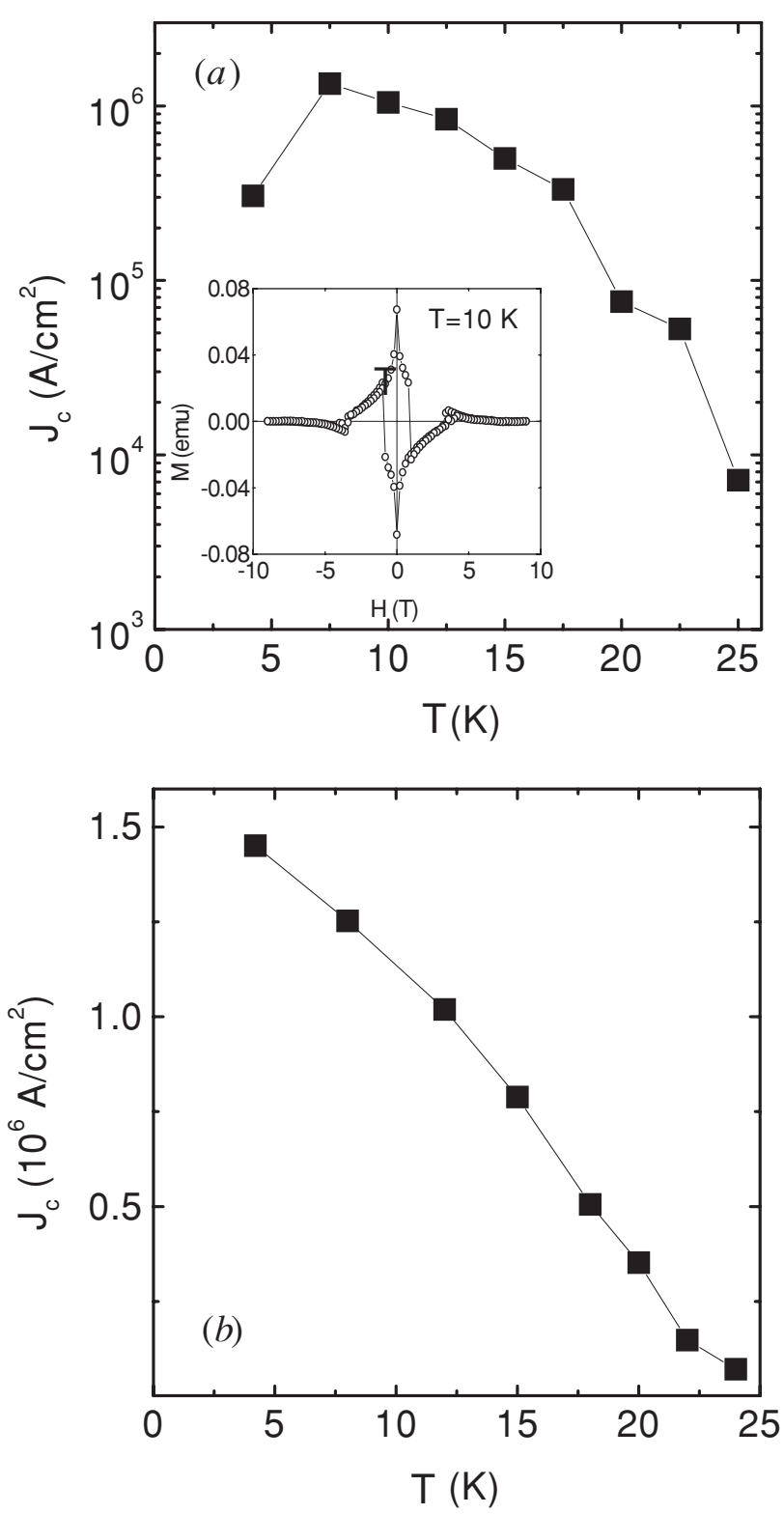

Figure 4. The temperature dependence of the zero-field $J_{\mathrm{c}}$ of a $4000 \AA$ thick $\mathrm{MgB}_{2}$ film. The inset shows the $M-H$ loop at $T=10 \mathrm{~K}$.

of $\mathrm{MgO}$ at the grain boundaries will act as series-connected resistors to the $\mathrm{MgB}_{2}$ grains. The superconducting transition temperature of the film, characterized by the zero resistance temperatures, is $34 \mathrm{~K}$. The superconducting transition is further characterized by the ac susceptibility, the result of which is shown in figure $3(b)$. The transition is relatively sharp with a full width at the half maximum of the imaginarypart signal being $\sim 0.8 \mathrm{~K}$.

The critical current densities of the $\mathrm{MgB}_{2}$ films were determined using both the magnetization and transport methods. In figure 4(a), the temperature dependence of $J_{\mathrm{c}}$ is plotted for a $4000 \AA$ thick $\mathrm{MgB}_{2}$ film. A zero-field $J_{\mathrm{c}} \sim 1.34 \times 10^{6} \mathrm{~A} \mathrm{~cm}^{-2}$ was obtained at $7.5 \mathrm{~K}$. The $M-H$ loop at $10 \mathrm{~K}$ for magnetic field $\mathbf{H} \perp$ film surface, which is $5 \mathrm{~mm} \times 4 \mathrm{~mm}$ in size, is shown in the inset. It shows a severe instability in flux pinning or flux jump, which causes the collapse of circulating critical current so that the magnetization 


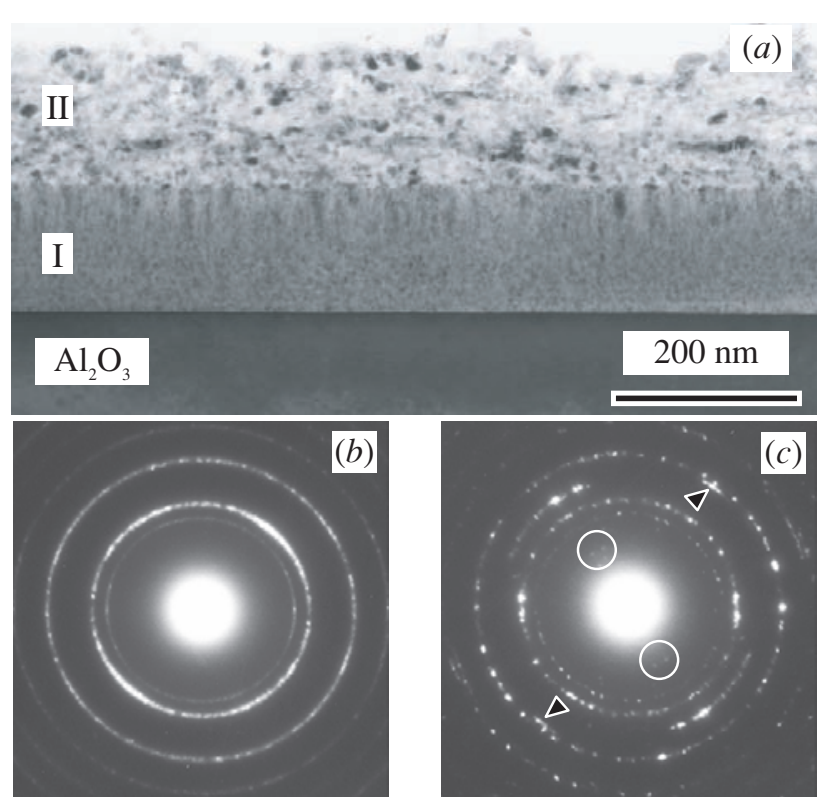

Figure 5. (a) Dark-field TEM image showing a cross-sectional view of a $\mathrm{MgB}_{2}$ thin film with $T_{\mathrm{c}}=32 \mathrm{~K}$. Selected-area electron diffraction patterns from $(b)$ region I and $(c)$ region II.

curve returns to the reversible magnetization branch (in the II and IV quadrants). The actual closing of the hysteresis curve excluding the flux jump occurs at a field of $\sim 8 \mathrm{~T}$ at low temperatures, suggesting an irreversibility field similar to that found in bulk $\mathrm{MgB}_{2}$ [6]. The temperature dependence of the transport $J_{\mathrm{c}}$ is plotted in figure $4(b)$ for a $2500 \AA$ thick $\mathrm{MgB}_{2}$ film. A narrow bridge with a width of $7 \mu \mathrm{m}$ was photolithographically patterned and the electrodes were formed by pressed indium. A criterion of $1 \mu \mathrm{V}$ was used to determine the critical current. As shown in the figure, a $J_{\mathrm{c}}$ value of $1.4 \times 10^{6} \mathrm{~A} \mathrm{~cm}^{-2}$ was obtained at low temperatures.

Compared to the early reports of in situ $\mathrm{MgB}_{2}$ thin films [8, 13-15] where $T_{\mathrm{c}}$ is around or below $25 \mathrm{~K}$, the $T_{\mathrm{c}}$ value shown here is much higher. The reason may be due to the different microstructures in these films. We have studied the structure of our films by both $\mathrm{x}$-ray diffraction and crosssectional transmission electron microscopy (TEM). In contrast to ex situ annealed $\mathrm{MgB}_{2}$ films with bulk-like $T_{\mathrm{c}}$ of $39 \mathrm{~K}$, which has a $\mathrm{MgB}_{2}$ grain size of $\sim 100 \AA$ and clear $\mathrm{x}$-ray diffraction peaks $[11,10]$, x-ray diffraction scans of our in situ annealed films revealed no discernable film peaks, indicating that the grain size was appreciably smaller. The correlation between the $\mathrm{MgB}_{2}$ grain size and superconducting properties is further corroborated by the TEM results of two $\mathrm{MgB}_{2}$ films of different $T_{\mathrm{c}}$ values.

The TEM result for a $\mathrm{MgB}_{2}$ film with $T_{\mathrm{c}}=32 \mathrm{~K}$ is shown in figure 5, and its $R$ versus $T$ curve is shown in figure 6 . The dark-field image in figure 5(a) shows that the film consists of two layers with different contrast. Figure $5(b)$ is a selectedarea electron diffraction pattern taken from region I near the film/substrate interface. By measuring the position and intensity distribution of the diffraction rings, it is determined that they all belong to the rock salt $\mathrm{MgO}$ phase. Figure 5(c) is a diffraction pattern taken from region II close to the film surface using the same size selected-area aperture as for figure $5(b)$. In addition to the diffraction rings corresponding to

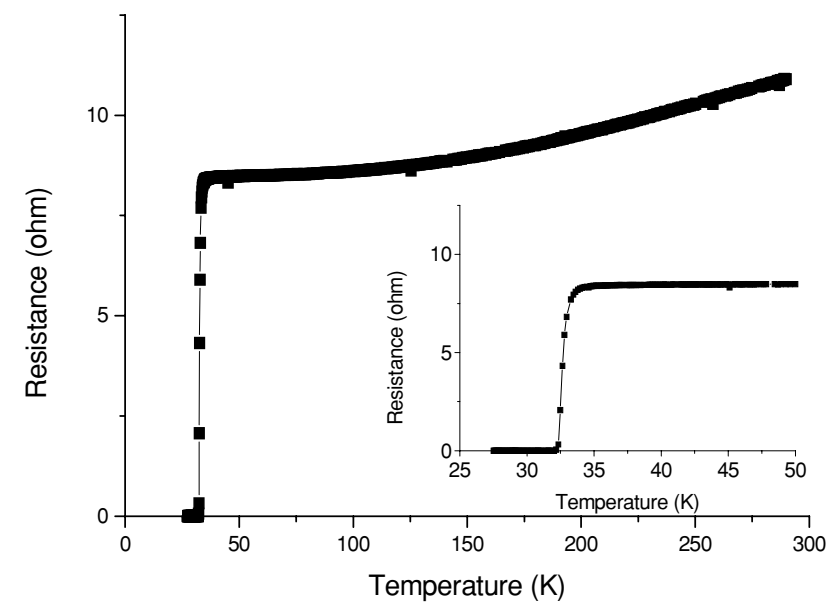

Figure 6. Resistance versus temperature curve for the film in figure 5 .

the $\mathrm{MgO}$ phase, it also shows diffraction spots corresponding to the hexagonal $\mathrm{MgB}_{2}$ phase. The diffraction spots indicated by the circles are due to the (001) planes, while those by the arrow heads arise from diffraction by the (110) planes of $\mathrm{MgB}_{2}$. The $(021) \mathrm{MgB}_{2}$ reflections are also detected. The discrete spots appear in figure $5(c)$ instead of nearly continuous rings in figure $5(b)$, indicating a larger grain size in region II. In both regions, the result indicates substantial oxygen contamination. The $\mathrm{MgB}_{2}$ grain size in region I must be less than about $50 \AA$ to account for the absence of $\mathrm{MgB}_{2}$ rings or spots in figure $5(b)$.

The TEM result for a second $\mathrm{MgB}_{2}$ film with $T_{\mathrm{c}}=21 \mathrm{~K}$ is shown in figure 7, and its $R$ versus $T$ curve is shown in figure 8 . From the dark-field image in figure $7(a)$ one can see that region $\mathrm{I}$ is thinner than that in the higher $T_{\mathrm{c}}$ film. As shown in figure $7(b)$, in both regions I and II, the $\mathrm{MgB}_{2}$ diffraction patterns were absent. The absence of the $\mathrm{MgB}_{2}$ patterns indicates that the $\mathrm{MgB}_{2}$ grain size in this film is less than $50 \AA$. When the grain size is close to the coherence length of $\mathrm{MgB}_{2}$, the superconducting properties will be affected. While the $900{ }^{\circ} \mathrm{C}$ annealing in the ex situ process provides enough thermal energy for crystallization and texturing, the lower temperature during the in situ annealing limits the extent of these processes. The reason that the $T_{\mathrm{c}}$ value reported here is much higher than those in the early reports of in situ $\mathrm{MgB}_{2}$ thin films [8, 13-15] may be that the grain size in our film is larger due to the details of the processing conditions.

The results of both figures 5 and 7 show severe oxygen contamination in the films. The diffraction patterns from $\mathrm{MgO}$ dominate both images. A separate XPS measurement of two films with different $T_{\mathrm{c}}, 17$ and $32 \mathrm{~K}$, respectively, shows that there is no appreciable difference in the oxygen content between the two films, and the $\mathrm{Mg}: \mathrm{B}: \mathrm{O}$ atomic ratio is 1.0:1.1:1.2. The extent of the detrimental effects of oxygen contamination is not well understood, and Eom et al even suggest that it may help to enhance the flux pinning [11].

\section{Conclusion}

In order to realize the potential of $\mathrm{MgB}_{2}$ in superconducting digital applications, epitaxial or polycrystalline film produced 

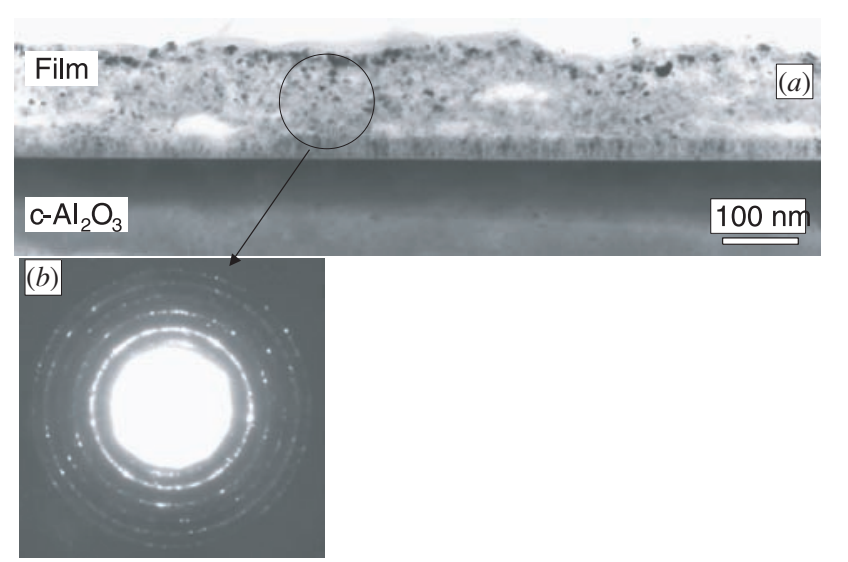

Figure 7. (a) Dark-field TEM image showing a cross-sectional view of a second $\mathrm{MgB}_{2}$ thin film with $T_{\mathrm{c}}=21 \mathrm{~K}$. (b) Selected-area electron diffraction pattern.

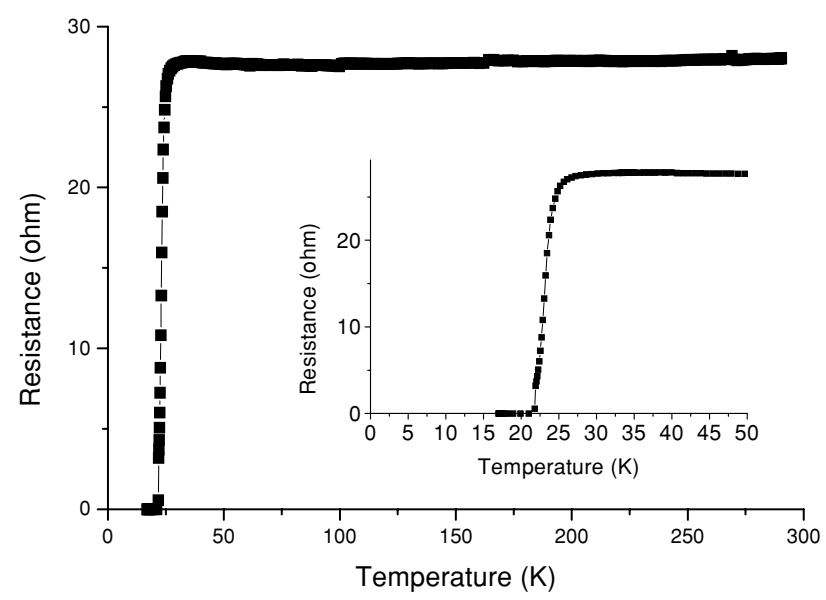

Figure 8. Resistance versus temperature curve for the film in figure 7.

by a processing technique compatible with multilayer depositions is needed. Using the CALPHAD technique we find that $\mathrm{MgB}_{2}$ is thermodynamically stable only under fairly high to very high $\mathrm{Mg}$ partial pressures at the temperature range appropriate for epitaxial growth. Therefore, a large $\mathrm{Mg}$ flux must be delivered from the deposition source for in situ $\mathrm{MgB}_{2}$ film deposition. This requirement favours deposition techniques that can maintain a large $\mathrm{Mg}$ flux over those where a large $\mathrm{Mg}$ flux is impractical. At present, no report of such deposition technique exists.

Lacking an in situ $\mathrm{MgB}_{2}$ film deposition technique, we have deposited $\mathrm{MgB}_{2}$ thin films by pulsed laser deposition using an in situ annealing process. The $T_{\mathrm{c} 0}$ obtained is much higher than those previously reported in situ films and $J_{\mathrm{c}}$ is comparable to those of the polycrystalline bulk samples even though the grain size in the films is extremely small. Because this deposition process is more compatible with multilayer deposition, it is important to demonstrate that high $T_{\mathrm{c} 0}$ and $J_{\mathrm{c}}$ can be obtained using this process. Our results are a step towards making the in situ annealing technique a viable candidate for $\mathrm{MgB}_{2}$ Josephson junction technologies. However, because the formation and crystallization of the $\mathrm{MgB}_{2}$ phase is constrained by the balance of several processes and by thermodynamic phase stability conditions and kinetics, it is difficult to achieve films with large grain sizes. An in situ film deposition technique is much more desirable for the $\mathrm{MgB}_{2}$ Josephson junction technologies.

\section{Acknowledgments}

This work is supported in part by ONR under grant no N0001400-1-0294 (XXX), by NSF under grant nos DMR-9875405 and DMR-9871177 (XQP), DMR-9876266 and DMR-9972973 (QL), DMR-9983532 (ZKL), and by DOE through grant DEFG02-97ER45638 (DGS).

\section{References}

[1] Nagamatsu J, Nakagawa N, Muranaka T, Zenitani Y and Akimitsu J 2001 Nature $\mathbf{4 1 0} 63$

[2] Cava R J 2001 Nature $4 \mathbf{1 0} 23$

[3] Bud'ko S L, Lapertot G, Petrovic C, Cunningham C E, Anderson N and Canfield P C 2001 Phys. Rev. Lett. 861877

[4] Karapetrov G, Iavarone M, Kwok W K, Crabtree G W and Hinks D G 2001 Phys. Rev. Lett. 864374

[5] Finnemore D K, Ostenson J E, Bud'ko S L, Lapertot G and Canfield P C 2001 Phys. Rev. Lett. 862420

[6] Larbalestier D C et al 2001 Nature 410186

[7] Bugoslavsky Y, Perkins G K, Qi X, Cohen L F and Caplin A D 2001 Nature 410563

[8] Shinde S R, Ogale S B, Greene R L, Venkatesan T, Canfield P C, Bud'ko S, Lapertot G and Petrovic C 2001 Appl. Phys. Lett. 79227

[9] Zhai H Y, Christen H M, Zhang L, Paranthaman M, Cantoni C, Sales B C, Fleming P H, Christen D K and Lowndes D H 2001 Preprint cond-mat/0103618

[10] Kang W N, Kim H-J, Choi E-M, Jung C U and Lee S-I 2001 Science 2921521

[11] Eom C B et al 2001 Nature 411558

[12] Moon S H, Yun J H, Lee H N, Kye J I, Kim H G, Chung W and Oh B 2001 Appl. Phys. Lett. 792429

[13] Blank D H A, Hilgenkamp H, Brinkman A, Mijatovic D, Rijnders G and Rogalla H 2001 Appl. Phys. Lett. 79394

[14] Christen H, Zhai H, Cantoni C, Paranthaman M, Sales B, Rouleau C, Norton D, Christen D and Lowndes D 2001 Physica C $\mathbf{3 5 3} 157$

[15] Grassano G, Ramadan W, Ferrando V, Bellingeri E, Marré D, Ferdeghini C, Grasso G, Putti M, Siri A S, Manfrinetti P, Palenzona A and Chincarini A 2001 Supercond. Sci. Technol. 14 762-4

[16] Heckingbottom R, Davies G and Prior K 1983 Surf. Sci. 132 375

[17] Seki H and Koukitu A 1986 J. Cryst. Growth 78342

[18] Hammond R H and Bormann R 1989 Physica C 162703

[19] Tsao J Y 1991 J. Cryst. Growth 110595

[20] Theis C D, Yeh J, Schlom D G, Hawley M E and Brown G W 1998 Thin Solid Films 325107

[21] Jones M and Marsh R 1954 J. Amer. Chem. Soc. 761434

[22] Massalski T (ed) 1990 Binary Alloy Phase Diagrams 2nd edn (Materials Park, OH: ASM International)

[23] Saunders N and Miodownik A P 1998 CALPHAD (Calculation of Phase Diagrams): A Comprehensive Guide (Oxford: Pergamon)

[24] Dinsdale A T 1991 CALPHAD, Comput. Coupling Phase Diagr. Thermochem. 15317

[25] Jansson B, Jonsson B, Sundman B and Agren J 1993 Thermochimica Acta 21493

[26] Nayeb-Hashemi A A and Clark J B 1988 Phase Diagrams of Binary Magnesium Alloys (Meterials Park, OH: ASM International)

[27] Yang M H and Flynn C P 1989 Phys. Rev. Lett. 622476

[28] Flynn C P 1988 J. Phys. F 18 L195 
[29] Loeb L B 1934 The Kinetic Theory of Gases (New York: McGraw-Hill) pp 19-106

[30] Schlom D and Harris Jr J 1995 Molecular Beam Epitaxy: Applications to Key Materials ed R Farrow (Park Ridge: Noyes) p 505

[31] Tsao J Y 1993 Materials Fundamentals of Molecular Beam Epitaxy (Boston: Academic)
[32] Fan Z Y, Hinks D G, Newman N and Rowell J M 2001 Appl. Phys. Lett. 7987

[33] Liu Z K, Schlom D G, Li Q and Xi X X 2001 Appl. Phys. Lett. 783678

[34] Canfield P C, Finnemore D K, Bud'ko S L, Ostenson J E, Lapertot G, Cunningham C E and Petrovic C 2001 Phys. Rev. Lett. 862423 